\title{
Effects of stacking faults on the electronic structures of quantum rods
}

\author{
Lin-Wang Wang \\ Computational Research Division \\ Lawrence Berkeley National Laboratory \\ 1 Cyclotron Road \\ Berkeley, CA 94720 \\ Phone: 510-486-5571 \\ Email: 1wwang@lbl.gov
}

\begin{abstract}
: atomistic semiempirical pseudopotential method is used to study the effects of stacking faults in a wurtzite structure quantum rod. It is found that a single stacking fault can cause a 10-50 meV change in the conduction state eigen energy, and a localization in the electron wavefunction. However, the effects on the hole eigen energies and wavefunctions are very small.
\end{abstract}

Key Words: nanorod, stacking fault, CdSe, empirical pseudopotential 
Colloidal semiconductor quantum dots synthesized by wet chemistry methods are one of the most well controlled nanosystems [1]. Not only their sizes can be controlled within $5 \%$ [2], they can also be synthesized into different shapes [3], and consisted of several semiconductor materials [4]. The optical spectroscopies of such nanosystems are well studied, and their electronic structures are relatively well understood [5]. Despite of all these controllability, stacking fault inside the nanostructure is a common feature. This can happen in wurtzite (WZ) or zinc blind (ZB) crystal structure quantum dots, like CdSe and InP. The stacking fault can be shown in Transmission Electron Microscope images [6]. The appearance of the stacking fault is probably due to the small energy differences between different stacking (e.g., the energy difference between WZ and ZB is only about $1 \mathrm{meV} /$ atom in CdSe [7]), and it depends sensitively on the growth kinetics in the synthesis process.

Despite the often existence of the stacking fault, theoretical comparison with experiments are often done using calculations based on pure $\mathrm{WZ}$ or ZB structures without the consideration of the stacking faults $[5,8]$. This raises the question: how large is the effects of stacking faults in the electronic structure of a quantum dot. This is an important question given the often existence of the stacking faults in the nanosystems. Here we use a well tested, atomistic semiempirical pseudopotential method (SEPM) [9] to address this question.

We choose a CdSe quantum rod as a representative system. Not only this system can be synthesized experimentally [3] and the existence of the stacking faults are often observed, its prolonged shape also makes it easy for the stacking fault study. The 
quantum rod we choose has a length of $7.5 \mathrm{~nm}$, and a diameter of $2.8 \mathrm{~nm}$. The total number of $\mathrm{Cd}$ and $\mathrm{Se}$ atoms is about 1500 . Its normal crystal structure is WZ. Using the terminology of a close pack metallic system, in the lateral plane perpendicular to the WZ c-axis [or the ZB (111) direction], there are three possible stacking positions $\mathrm{A}, \mathrm{B}, \mathrm{C}$ to specify a packing plane. In our tetrahedral semiconductor, one packing plane is consisted of two atomic monolayers, one Se layer and one Cd layer at the same atomic lateral position. Using this notation, any sequence $\mathrm{ABCBACB}$... will represent a tetrahedral structure as long as no two adjacent layers are the same (i.e, no AA, BB, or CC). Then, as well known, a WZ structure is ABABABABAB... and a $\mathrm{ZB}$ structure is ABCABCABCABC.... Given the quantum rod length we have chosen above, there will be 22 packing planes in the packing sequence of one quantum rod. To study the effect of a single stacking fault, we will start with a WZ sequence [to be called sequence (a)]: $\mathrm{ABABABABAB} . .$. , and introduce a stacking fault in the middle, then to calculate the changes in the resulting electronic structures.

There are many ways to introduce a single stacking fault in the middle of a WZ sequence. They can be summarized as following: sequence b: ABABABCACACA; sequence c: ABABABCBCBCB; sequence d: $\mathrm{ABABABCABABAB}$; sequence e: ABABABCBABABA. After the atomic structures are determined, the electronic structure calculations will be carried out using semiempirical pseudopotentials [9]. These pseudopotentials $v_{\alpha}(r)$ are fitted to the local density approximation (LDA) total screened potentials of different crystal structures, and experimental bulk band structures of WZ CdSe. They have local parts and nonlocal parts. They include spin-orbit interactions. The total potential of a nanosystem is simply a sum of these atomic semiempirical 
pseudopotentials: $\sum_{R} v_{\alpha}(r-R)$, here $\mathrm{R}$ is the atomic positions. The electron wave functions $\psi_{i}(r)$ are expanded by planewave basis. A 6.9 Ryd kinetic energy cutoff is used to define this planewave basis set. The single particle Schrodinger's equation $\left[-\frac{1}{2} \nabla^{2}+\sum_{R} v_{R}(r-R)\right] \psi_{i}(r)=E_{i} \psi_{i}(r)$ is solved for its eigen states $\psi_{i}(r)$ near the band gap using the folded spectrum method $[10,11]$. In the fitted bulk band structures, of interest here is the band offsets between the WZ and ZB crystal structures. In our SEPM, we have $E_{C B M}(W Z)-E_{C B M}(Z B)=144 \mathrm{meV}$, and $E_{V B M}(W Z)-E_{V B M}(Z B)=59 \mathrm{meV}$, here CBM stands for conduction band minimum, VBM stands for valence band maximum. These band offsets are in good agreements with previous ab initio calculations [7, 12, 13] and experimental results [14].

Figure 1 shows the atomic structure and the calculated electron state and hole state of a perfect $\mathrm{WZ}$ structure quantum rod. From the wavefunction charge density plots in Figure 1, we can see that the hole state has a nodal plane between two adjacent stacking planes, while the electron states are connected between the planes in a more complicated way. For the hole state, each stacking plane looks independent. Thus a change of the stacking sequence might have a minimum consequence on the hole wavefunction. This is confirmed by looking at Figure 2, where the electron and hole state wavefunctions of a $\mathrm{ZB}$ structure quantum rod are shown. The $\mathrm{ZB}$ structure has an $\mathrm{ABCABCABC}$ sequence (to be called sequence f). But the hole state looks very much alike the hole state in the WZ structure. This is not true for the electron states. Despite some similarities, the difference between the electron state in $\mathrm{WZ}$ and in $\mathrm{ZB}$ are quite significant. This suggests that a stacking fault will have a bigger effect on the electron state. 
The wavefunctions of the quantum rods with one single stacking fault are plotted in Figures 3 and 4 . What plotted there is a planar average of the wavefunction square in the lateral direction. The electron state eigen energies are $-2.452,-2.436,-2.465,-2.451 \mathrm{eV}$ for $b, c, d$, e stacking fault sequences respectively. They should be compared with the original electron eigen energy of $-2.426 \mathrm{eV}$ of the pure $\mathrm{WZ}$ quantum rod (sequence a). Also, in comparison, the electron eigen energy of the pure ZB quantum rod (sequence f) shown in Fig.2(a) is $-2.560 \mathrm{eV}$. We see that, compared to the original WZ structure, a single stacking fault causes an energy drop in the order of 10-40 meV. The electron energy difference between the $\mathrm{ZB}$ and $\mathrm{WZ}$ quantum rods is $134 \mathrm{meV}$, very close to the bulk CBM difference $144 \mathrm{meV}$. The bulk WZ CBM has a energy of $-2.683 \mathrm{eV}$, thus the electron quantum confinement energy for the $\mathrm{WZ}$ quantum rod is $257 \mathrm{meV}$. The stacking fault caused energy change in the quantum rod is relatively small compared to this quantum confinement energy. This means that previous theoretical calculations without stacking faults are probably okay in deducing the quantum confinement energies. By looking at the wavefunction plots in Fig.3, we see that all the stacking faults have caused some degree of localizations. For one dimensional system, it is known that any shallow potential well will cause a localized bound state. Since all the stacking fault systems have lower energy electron states than the pure WZ quantum, we can thus deduce that for an infinitely long WZ quantum wire, a single stacking fault will cause a localized electron bound state.

It is interesting to compare the 4 stacking faults we have calculated. Sequence $d$ has the lowest energy and the strongest localization. Part of the reason is probably because this sequence has a segment "ABCAB" at the stacking fault region which resembles a $\mathrm{ZB}$ 
structure, and this segment is the longest among the 4 stacking fault structures we have studied here. Another question one can ask is that whether the long range ordering plays any role in the electron energy and its localization. For example, in sequence $\mathrm{d}$ and e, both the right hand side and left hand side have the sequences: $\mathrm{ABABAB}$, thus we can say they have a long range ordering. On the other hand, for sequence b, the left hand side is: $\mathrm{ABABAB}$, while the right hand side is $\mathrm{ACACAC}$. The situation is similar for sequence $\mathrm{c}$. For these cases, we can say they no longer have the long range ordering. The long range ordering is a concern under some model considerations. For example, using a simple effective mass model, the left hand side and the right hand side wavefunctions are connected by a thin perturbative stacking fault region. Then, the Bloch part $\mathrm{u}(\mathrm{r})$ of the wavefunction from the two sides will be the same under long range ordering ( $\mathrm{d}$ and $\mathrm{e}$ ), and will be dislocated in the cases of $\mathrm{b}$ and $\mathrm{c}$. From our calculation, there is no strong evidence showing that the long range ordering is playing a significant role in the resulting electronic structures. Thus, in summary, the electronic structure is affected mostly by the atomic structures near the stacking fault, not by far away long rang ordering.

In striking contrast to the electron states shown in Fig.3, the hole wavefunctions shown in Fig.4 for different stacking faults are almost indistinguishable from the original pure $\mathrm{WZ}$ quantum rod. The eigen energy differences from the original $\mathrm{WZ}$ quantum rod are within $7 \mathrm{meV}$, and for $\mathrm{b}$ and $\mathrm{d}$ this difference is only $1 \mathrm{meV}$. Compared to the bulk VBM, the hole state quantum confinement energy for the $\mathrm{WZ}$ quantum rod is $296 \mathrm{meV}$. The small effects on the hole states are related to the nodal structures of the hole wavefunctions as shown in Figures 1(b) ,2(b) and as discussed above. This planar nodal structure is also demonstrated in Fig.4 as the deep dips. Interestingly, the hole eigen 
energy of the pure $\mathrm{ZB}$ quantum rod is $-4.787 \mathrm{eV}$, only $11 \mathrm{meV}$ lower than the pure $\mathrm{WZ}$ quantum rod result. This difference is $59 \mathrm{meV}$ in the bulk. This means that the quantum confinement effect (especially from the lateral directions) has significantly reduced the valence band offset between the $\mathrm{WZ}$ and $\mathrm{ZB}$ quantum rods. This is different from the conduction band where the bulk band offset has been almost completely transferred into the electron energy difference in the quantum rods. Also interesting is that the hole eigen energies under the stacking faults are not within the $11 \mathrm{meV}$ energy window between the WZ quantum rod hole state and the ZB quantum rod hole state.

Note that the weak effects on the hole states can not be guessed from simple models. Although the bulk valence band offset is 2.5 times smaller than the bulk conduction band offset, but the light hole effective mass is 2 times larger than the electron effective mass, and the heavy hole effective mass is more than ten times larger than the electron effective mass. Thus, according to this simple effective mass picture, the effects on the hole state wavefunction should be similar to the electron wavefunctions. Also from previous calculations, we known that the hole state is easier to be localized than the electron state, for example under external electric fields [15]. One possible reason for the small hole state effects is that the stacking fault region (ZB like region) in $\mathrm{WZ}$ behaves as a potential barrier for the hole, not as a well. This is different from the electron situation. To test this, we have calculated a sequence g, which has a stacking fault (WZ like region) in a ZB

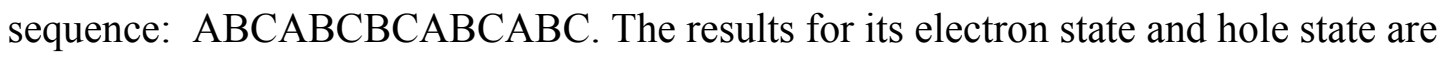
shown in Fig.5 and Fig.6, along with the original pure ZB quantum rod wavefunctions. Although in this case, the stacking fault region serves like a potential well for the hole state, the hole wavefunction still looks like the original ZB and WZ wavefunctions, 
doesn't show any significant localization. On the other hand, the electron wavefunction of this sequence $g$ shows a dip near the stacking fault, indicating the stacking fault region does behave like a potential barrier for the electron. Thus, in conclusion, the sign of the valence band offset is not the reason for the small stacking fault effects on the hole states. We just cannot use simple effective mass picture to understand the smallness of these effects.

The change of the wavefunction can also be measured by the change of oscillator strength: $\left\langle\psi_{C B M}|P| \psi_{V B M}\right\rangle^{2}$ where $\mathrm{P}$ is a momentum operator. This oscillator strength is related to physical observables like the photoluminescence life time. Compared to the pure WZ quantum rod, the single stacking fault quantum rods of sequences b,c,d,e have only changed this oscillator strength by $1-2 \%$. This small change is just a demonstration that the localization of the wavefunction has little effects on the oscillator strength, since it simply shifts the weight of the wavefunction from one place to another without affecting the product sum between the CBM and VBM states.

We have also calculated two random sequences, to be denoted as sequences $\mathrm{h}$ and $\mathrm{i}$. One can consider these two systems as consisted of many stacking faults. Their electron states and hole states are shown in Fig.5 and Fig.6 respectively. As we can see in Fig.5(d) for sequence $i$, the electron state wavefunction has been significantly changed from the pure $\mathrm{WZ}$ and $\mathrm{ZB}$ quantum rods. As for the eigen energies, the random sequence electron results are between the pure WZ and pure ZB quantum rod results. Similar to the single stacking fault situations, for the hole states, the random sequence quantum rod wavefunctions are almost the same as the $\mathrm{WZ}$ and $\mathrm{ZB}$ quantum rod wavefunctions, and their eigen energies differ only by a few meV. 
In summary, we find that a stacking fault can cause an eigen energy change of about $10-50 \mathrm{meV}$ in a quantum rod. The change comes mostly from the conduction band. The effect on the hole state is very small. This is because the hole wavefunction has a nodal structure between the stacking planes, while the electron wavefunctions are connected between the stacking planes. A single stacking fault in WZ will cause some degree of localization at the stacking fault for the electron wavefunctions. The changes of the wavefunctions and the eigen energies are determined mostly by the atomic arrangement near the stacking fault, not by the long range ordering. For a random stacking sequence, the electron wavefunction can be significantly altered, while the hole wavefunctions remain the same as in the pure $\mathrm{WZ}$ quantum rod.

This work was supported by U.S. Department of Energy under Contract No. DEAC03-76DF00098. This research used the resources of the National Energy Research Scientific Computing Center. 


\section{References}

1. A. P. Alivisatos, J. Phys. Chem. 100, 13226 (1996).

2. C. B. Murray, D. J. Norris, and M. G. Bawendi, J. Am. Chem.. Soc. 115, 8706 (1993).

3. L. Manna, E. C. Scher, and A. P. Alivisatos, J. Am. Chem. Soc. 122, 12700 (2000).

4. M. A. Hines, and P. J. Guyot-Sionnest, J. Phys. Chem. 100, 468 (1996).

5. L. W. Wang, and A. Zunger, J. Phys. Chem. B 102, 6449 (1998).

6. X. Peng, M. C. Schlamp, A. V. Kadavanich, and A. P. Alivisatos, J. Am. Chem. Soc. 119, 7019 (1997).

7. S.-H. Wei, and S. B. Zhang, Phys. Rev. B 62, 6944 (2000).

8. Al. L. Efros, M. Rosen, M. Kuno, M. Nirmal, D. J. Norris, and M. Bawendi, Phys. Rev. B 54, 4843 (1996).

9. L. W. Wang, and A. Zunger, Phys. Rev. B 51, 17398 (1995).

10. L. W. Wang, and A. Zunger, J. Chem. Phys. 100, 2394 (1994).

11. A. Canning, L. W. Wang, A. Williamson, A. Zunger, J. Comp. Phys. 160, 29 (2000).

12. C.-Y. Yeh, S.-H. Wei, and A. Zunger, Phys. Rev. B 50, 2715 (1994).

13. M. Murayama and T. Nakayama, Phys. Rev. B 49, 4710 (1994).

14. A. L. Edwards, and H. G. Drickamer, Phys. Rev. 122, 1149 (1961).

15. L. W. Wang, J. Phys. Chem. 105, 2360 (2001). 


\section{Figure Captions}

Figure 1. The isosurface plots of the charge densities (wavefunction square $\psi^{2}$ ) for conduction band minimum (CBM) (a) and valence band maximum (VBM) (b) states for a pure wurtzite structure quantum rod. The small balls represent the Se atoms. For clarity, $\mathrm{Cd}$ atoms are not shown. The horizontal direction is the c-axis of the wurtzite structure. The CBM isosurface value is $7 \times 10^{-5} \mathrm{e} / \mathrm{Bohr}^{3}$, while the VBM isosurface value is $8 \times 10^{-5}$ e/Bohr ${ }^{3}$.

Figure 2. The isosurface plots of the charge densities of CBM (a) and VBM (b) for a pure zinc blende structure quantum rod. The small balls represent the Se atoms. The horizontal direction is the zinc blende (111) direction. The CBM isosurface value is $7 \times 10^{-5} \mathrm{e} / \mathrm{Bohr}^{3}$, while the VBM isosurface value is $8 \times 10^{-5} \mathrm{e} / \mathrm{Bohr}^{3}$.

Figure 3. The planar averaged electron state charge densities $\left(\psi^{2}\right)$ plotted along the $\mathrm{z}$ direction (c-axis of the wurtzite structure). The stacking sequences are denoted as $\mathrm{ABC}$.. in the $\mathrm{x}$ axis. The numbers are the electron eigen energies. The sequence $\mathrm{a}$ is the same system as shown in Fig.1, while system b, c, d, e are single stacking fault sequences.

Figure 4. The planar averaged hole state charge densities plotted along the $\mathrm{z}$ direction. The systems are the same as in Fig.3.

Figure 5. The planar averaged electron state charge densities for (a): pure ZB structure; (b) a single stacking fault in the ZB sequence; (c) and (d) two random sequences. The sequence $f$ in (a) is the same system as shown in Fig.2. 
Figure 6. The planar averaged hole state charge densities for the same systems as shown in Fig.5. 
(a) $\mathrm{CBM}$

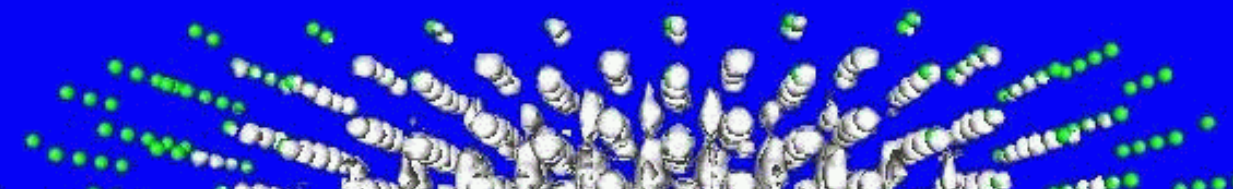

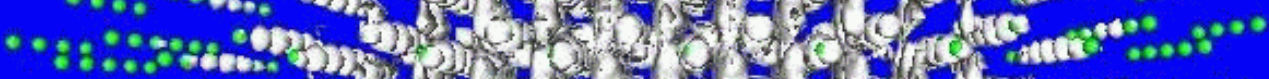

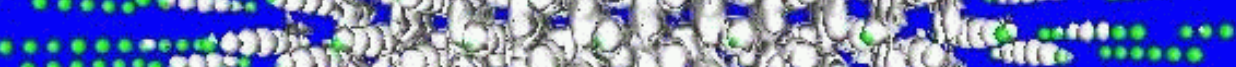

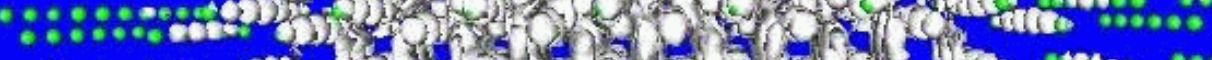

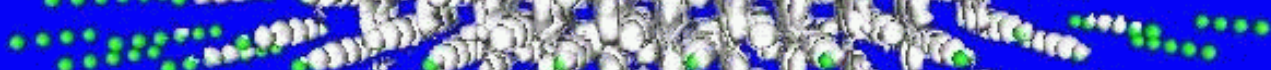

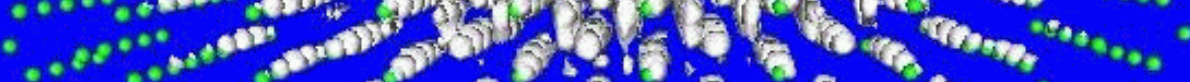

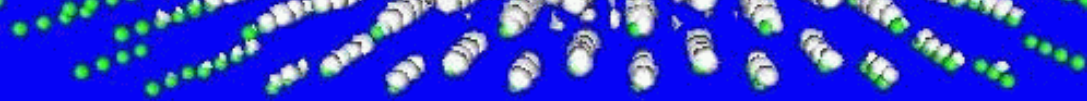

(b) VBM

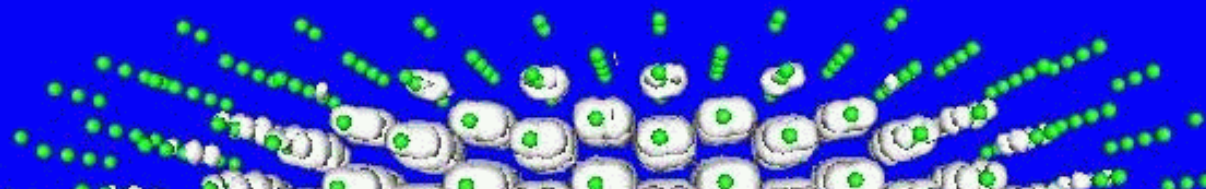

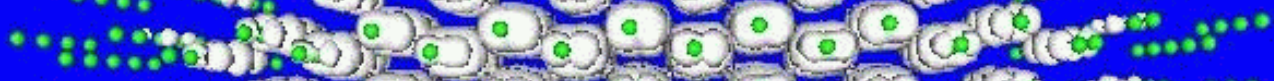

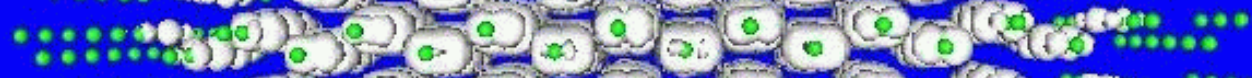

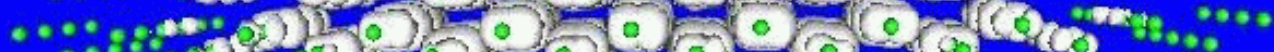

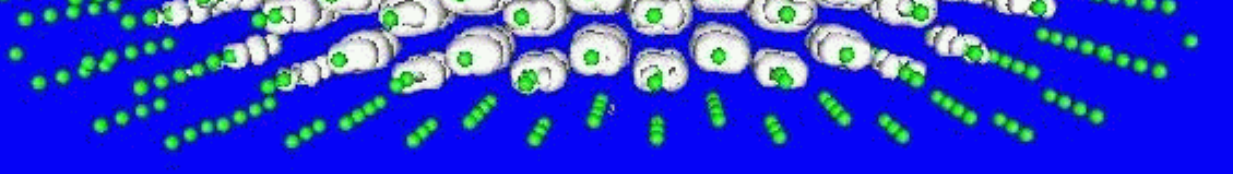

Fig.1, Wang 
(a) $\mathrm{CBM}$

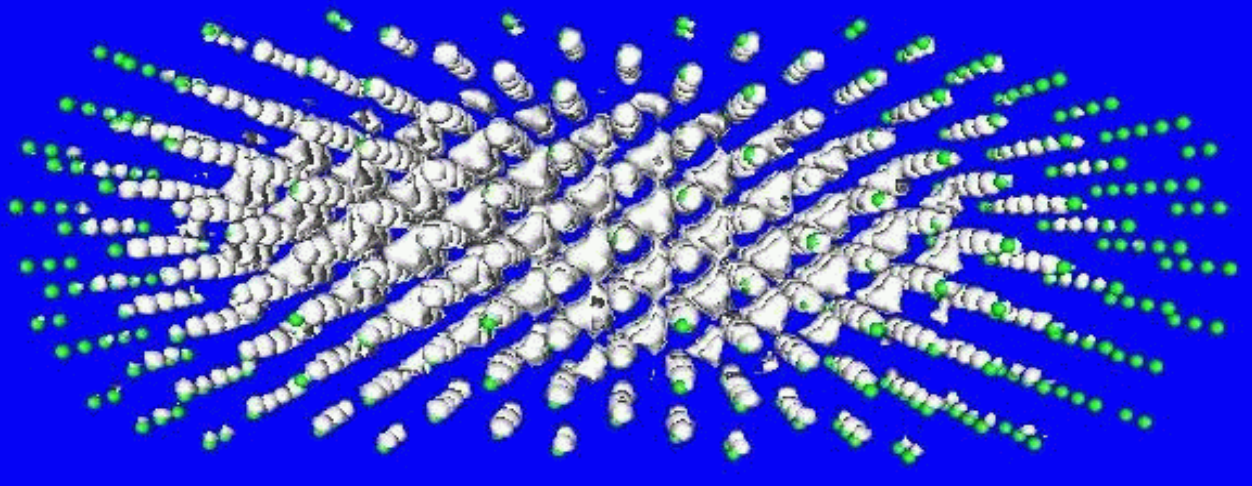

(b) VBM

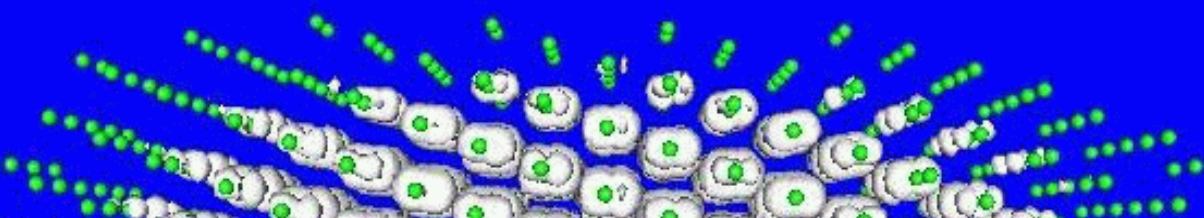

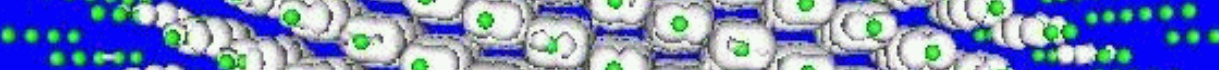

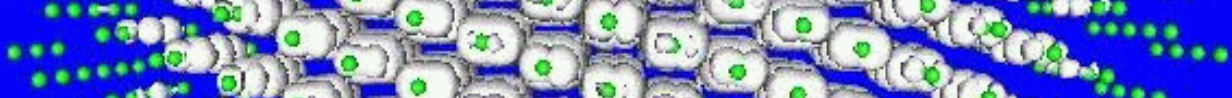

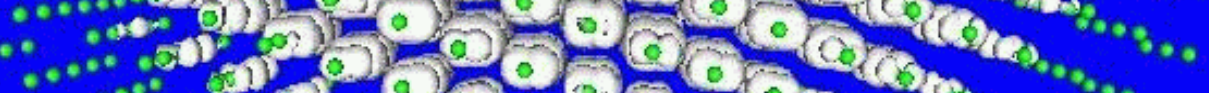

$$
\begin{aligned}
& \text {..... }
\end{aligned}
$$

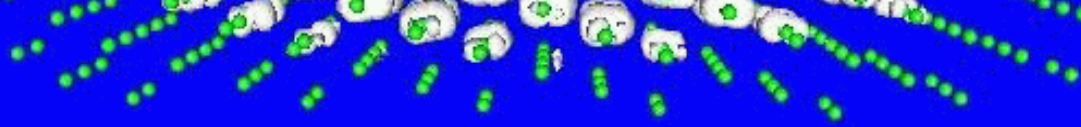

Fig.2, Wang 
Z position $(\stackrel{\AA}{A})$

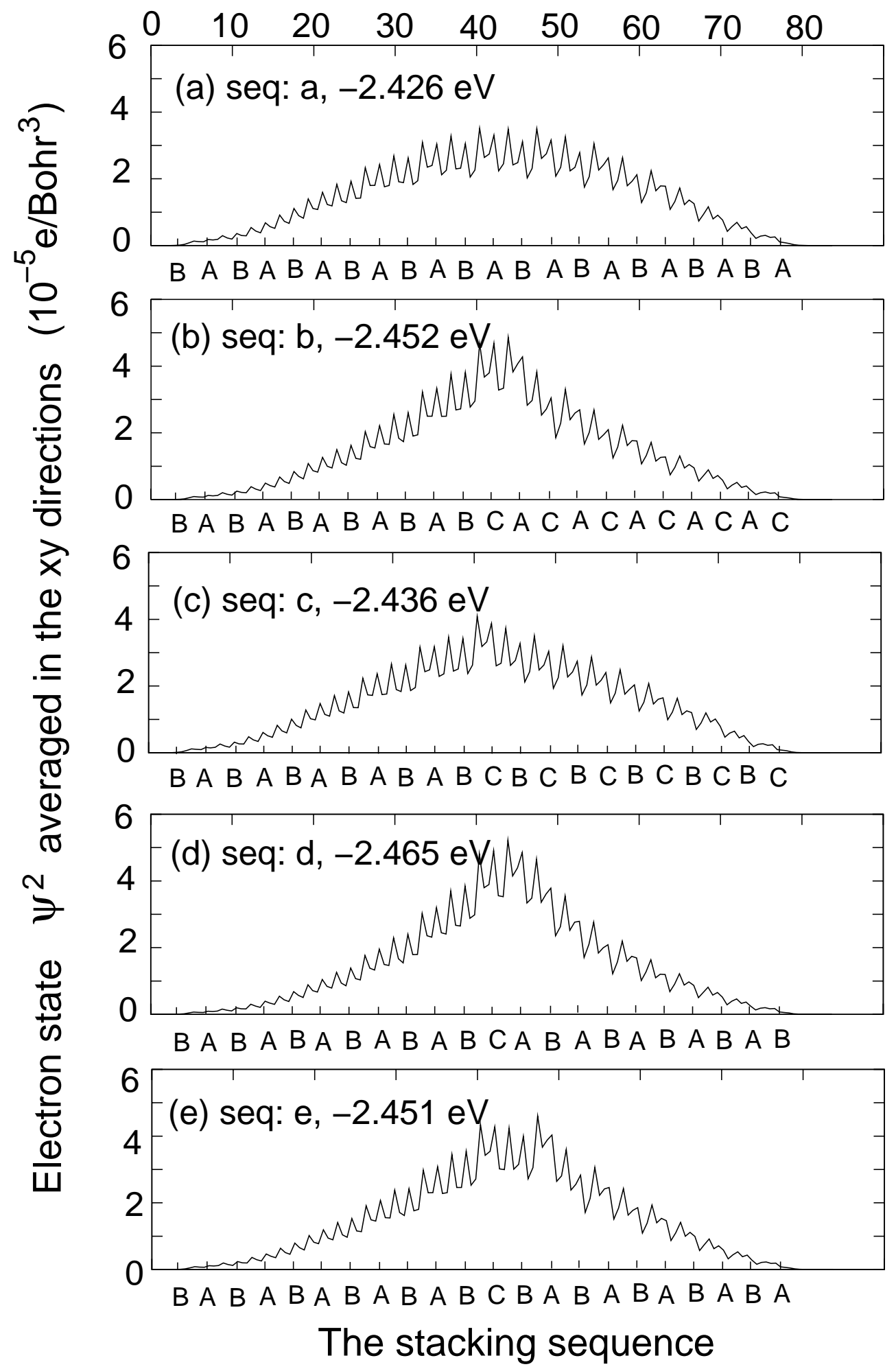

Fig.3, Wang 
Z position $(\stackrel{\AA}{)})$
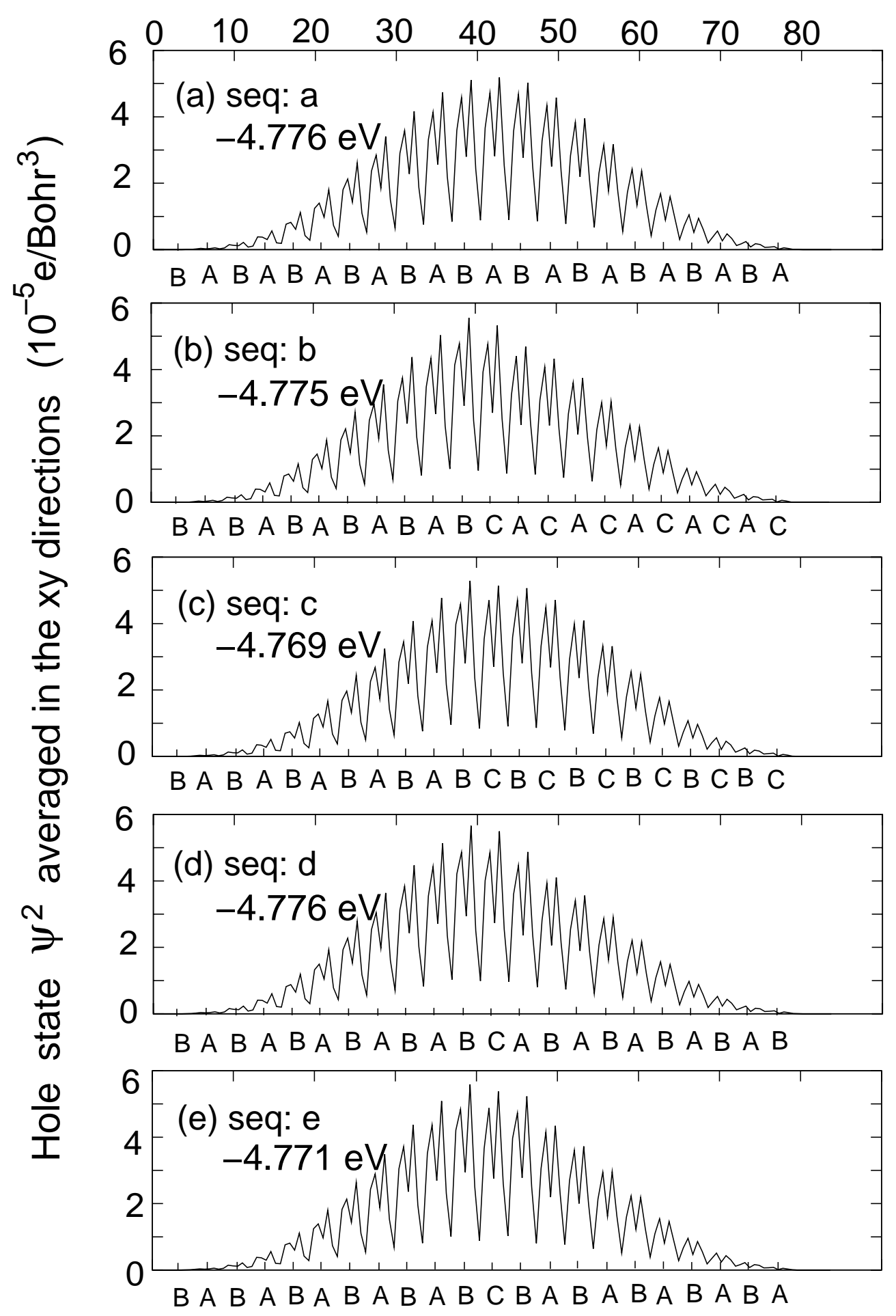

The stacking sequence

Fig.4, Wang 
Z position $(\stackrel{\wedge}{A})$
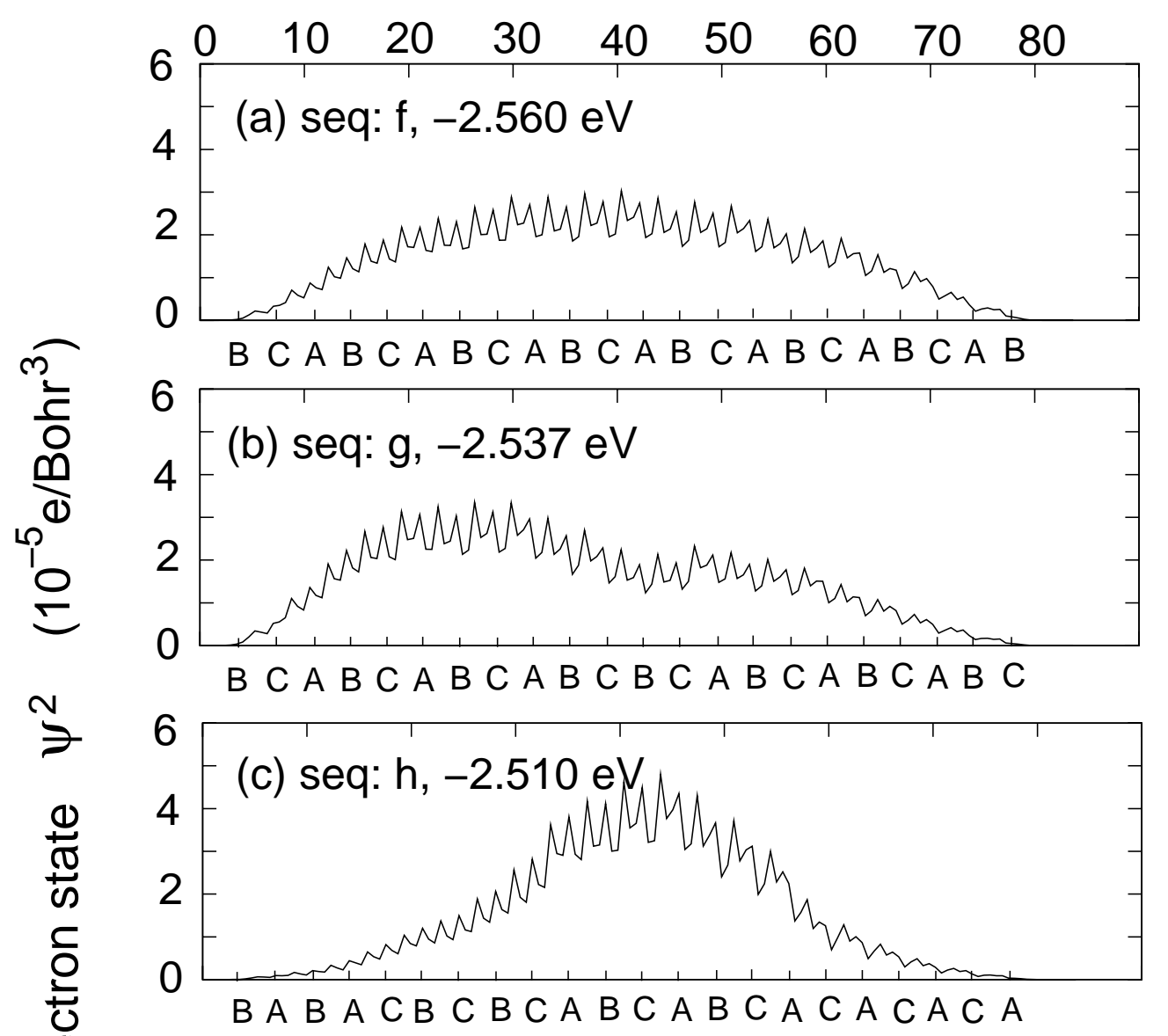

Ш

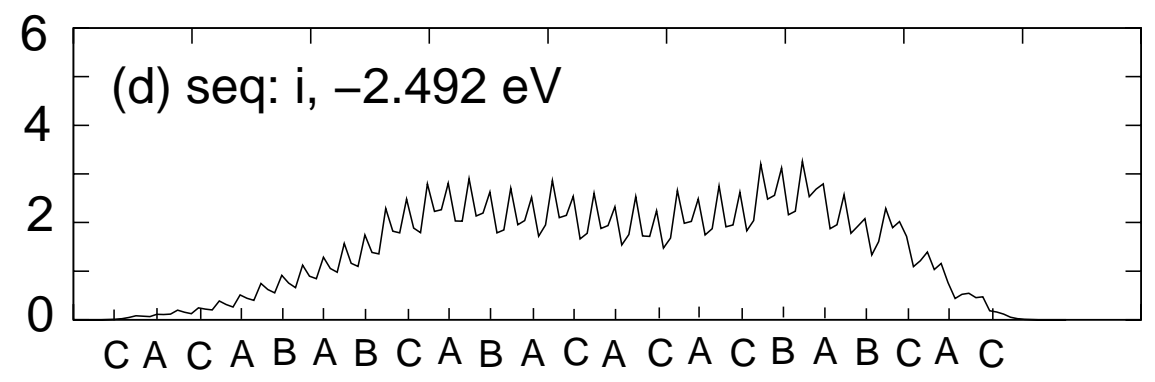

The stacking sequence

Fig.5, Wang 
Z position $(\stackrel{\circ}{A})$

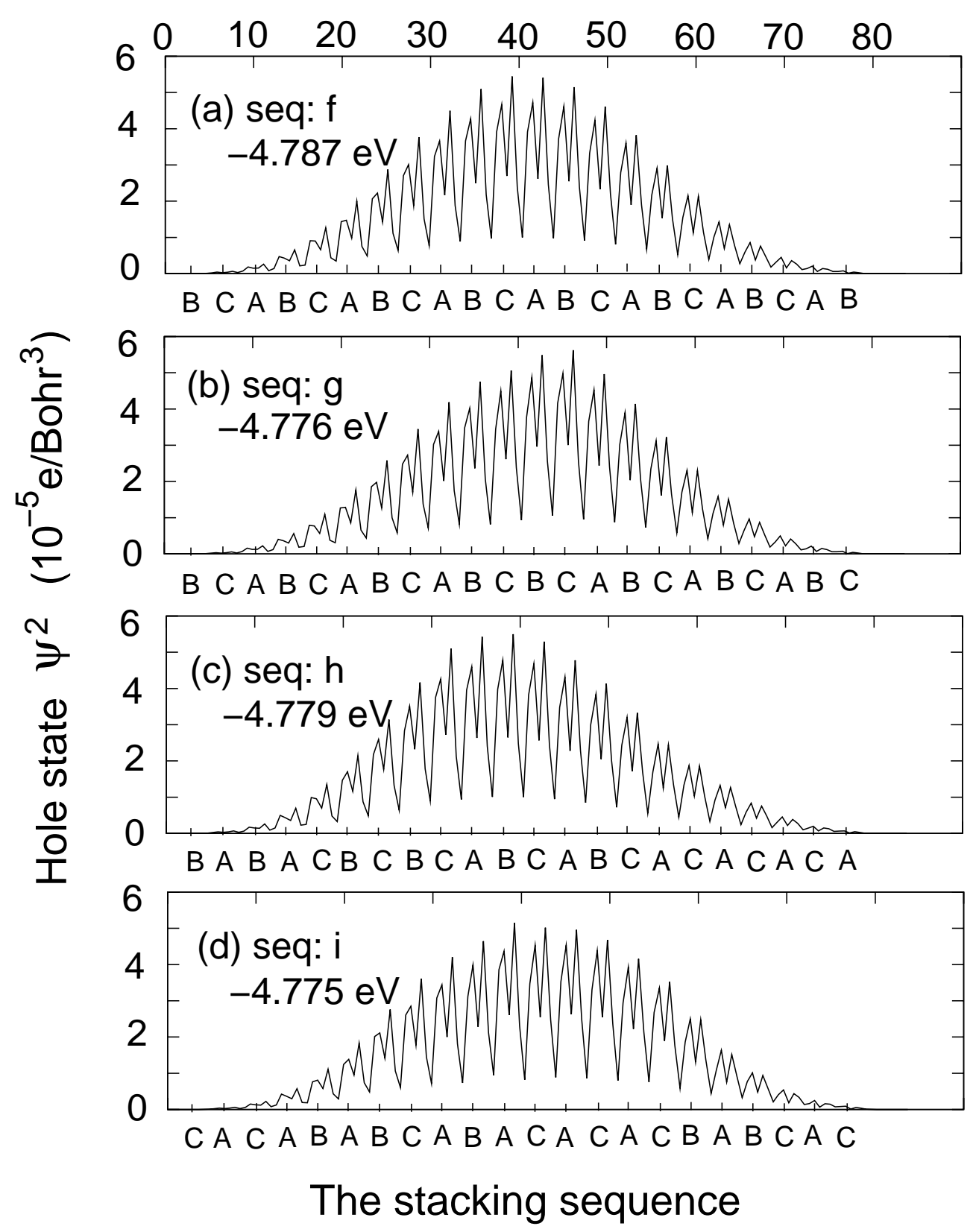

Fig.6, Wang 